\title{
Solar Panel Cells as Power Source and Li-Fi Data Nodes Integrated with Solar Concentrator
}

\author{
Siddharth Agarwal \\ Dept. of I.T. \\ Bharati Vidyapeeth Deemed \\ University, College of \\ Engineering, Pune, India
}

\author{
Yash Omer \\ Dept. of I.T. \\ Bharati Vidyapeeth Deemed \\ University, College of \\ Engineering, Pune, India
}

\author{
T.B. Patil \\ Dept. of I.T. \\ Bharati Vidyapeeth Deemed \\ University, College of \\ Engineering, Pune, India
}

\author{
Supriya C. Sawant \\ Dept. of Electronics \& \\ Telecommunication, Smt. \\ Kashibai Navale College of \\ Engineering, Pune, India
}

\begin{abstract}
The usage of internet and requirement for data transmission is at tremendous demand. People are more dependent on the internet compared to previous decades. So with the increase in number of users, spectrum is becoming congested. Presently Wi-Fi technology is popular which runs on radio frequency waves having less bandwidth so, to have a larger bandwidth for greater data transfer rate $\mathrm{Li}-\mathrm{Fi}$ has been introduced. $\mathrm{Li}-\mathrm{Fi}$ uses visible light spectrum having bandwidth 10,000 times more than radio waves. $\mathrm{Li}$-Fi requires continuous electric power for illumination to transfer data. Electrification is a major concern in rural areas. Uses of this Li-Fi technology with solar panel make it more efficient and easily available at every nook and corner. This paper deals with integration of $\mathrm{Li}-\mathrm{Fi}$ with solar panel. Solar panel is used instead of photodiodes for demodulation of data. Solar panels consist of solar cells which works for both storing energy and converting that energy stored in electrical signal for illuminating LED lamps and to complete the successful working of life technology also solar panel have also become more efficient by placing the solar concentrator above them which will increase the output power of solar panel manifold.
\end{abstract}

Keywords- c-Light fidelity (Li-Fi); Solar Panel; Fresnel Solar Concentrator.

\section{INTRODUCTION}

Today, the whole world is interlinked through the internet but still there some unprivileged sections, which are bereft, of the data services .At present people are familiar with Wi-Fi services, which is restricted to certain confined area, may be to office/campus etc. Wi-Fi having the speed for data transfer nearly 800 kbps-11mbps and moreover not so much securable. Therefore, to overcome the limitations new invention is done by a German scientist Harald Haas, which is known as Li-Fi, stand for light fidelity. Li-Fi runs in visible spectrum and is more efficient and easily available. Li-Fi consists of broad range of frequencies and wavelengths from the infrared through visible and finally down to the ultraviolet spectrum. LED light bulb is used to send human eyes cannot capture data

in Li-Fi whose intensity varies so faster that so the output appears to be constant. The working is based on the ON-OFF activities of LED. When the LED is ON, logically it represents the ' 1 ' is transmitted and when the LED is OFF, logically it represents the ' 0 ' is transmitted. Therefore, the data is transferred in digitalized format consisting of $0 \mathrm{~s}$ and $1 \mathrm{~s}$.at a very high speed and in a more securable way. data gets encoded at the transmitter section by switching system which is demodulated the receiving section Photodiodes are used as receivers for transmitting data to device .amplifiers are connected to photodiodes for removing interruptions in data. .Ten thousand times the data density of Wi-Fi can be achieved by $\mathrm{Li}-\mathrm{Fi}$ because of low interference in visible light spectra also Li-Fi requires fewer components and most important is there is no effect to human health by using this technology. LiFi can be used in many fields which includes medical field, airlines ,power plants and many more .Although in Li-Fi technology photodiodes which are used are very efficient in transmitting data still they are new technologies which has come in picture having lot of advantages over photodiodes that is the use of solar cells for demodulation of data. This techniques has been recently shown by Harald Haas to run LiFi with the use of solar panel .Solar panel also consist photovoltaic cells which are electrically connected which can be used to generate and supply electricity anywhere. Solar cell of photovoltaic cell converts the sun energy into electricity. Solar panels, consists of photovoltaic cells that changes incoming sunlight into electricity instead heat. Solar photovoltaic cells are made from silicon and consist of a positive and a negative film of silicon placed below thin slice of glass. Photons emitted by the sunlight strike down upon these cells, to knock the electrons off the silicon. The negatively charged free electrons are gathered to one side of the silicon cell, to create an electric voltage that can be collected and channeled. Solar photovoltaic array is formed by wiring together the solar panels where finally current is formed. Solar cells which are been used decodes the data sent by led lamp .Present paper deals with integration of LI-FI with solar panel which will enhance the Li-Fi technology and it will possible in to have the application of this technology at every corner of the world which will include all the rural density which are unaware of the technologies. Energy provides by sun is infinite it is just the lack of knowledge and skill, which make its use, 
limited. The further discussion_will clarify how to connect/integrate Li-fi with solar panel. .

\section{LITERATURE SURVEY}

Majority of people are using Wi-Fi Internet devices, where data is transmitted through wireless media access covering our home, offices, schools, and other public areas. Wi-Fi can cover an entire house, school, and its bandwidth is restricted to 50100 megabits per seconds (Mbps). Wi-Fi is insufficient for moving large data files like high definition movies, music libraries and video games. So Wi-Fi may not be enough as technology to meet the new demands so a new technology named as Li-Fi has been raised by a German scientist herald at the University of Edinburgh in 2011 where data is transmitted through the use of lights at a very high speed . LiFi is light-based communications technology that delivers a high-speed, bidirectional networked, mobile communications in a similar fashion as seen in Wi-Fi. Harald Haas has demonstrated the working of LI-FI technology with the use of led lamps and photodiode at ted global talk. [16] photodiode are used as the broadband the most efficient way of transmitting data without any damage to data.[17]But as we see recently herald has come up with some new adulterant in Li-Fi technology which is far beneficial for all and have lot of advantages. Before Li-Fi was been in use with photodiode for transmitting data but to overcome some of the limitation of photodiode herald at ted global talk held in 2015 talked about use of solar panel with Li-Fi[18].Solar panel is more beneficial it can perform both function of transmitting data and capturing energy whereas photodiode requires external device for the requirement of transmitting data Considering this new technology some more adulteration can be made to make it more efficient which is main concern of this paper by using solar concentrator .[19] Solar concentrator is a device that magnify the incoming solar radiation to increase the output power of solar panel.[20]Earlier as we have seen solar panel consist of solar cells which were used to coverts the radiation coming from sun into electricity but by the use of solar concentrator less solar cells will be required which will result in cost reduction and the efficiency will be far better as compared to previous case in which solar concentrator was not used with solar panel.

\section{PROPOSED SYSTEM}

B The system defined here, consist a led lamp, solar panel and solar concentrator, now all the data from Internet is streamed into lamp driver. Lamp driver will pass the data into led lamp, which will further send the encoded data to solar cells. Solar cell decodes encoded data transmitted by LED lamp. The data is encoded with sudden change of brightness of light signal. Solar concentrator is placed before solar panel, which will increase the intensity of solar cells rapidly so that the data can be transmitted for longer duration of time.

Solar concentrator is a device that magnifies the incoming solar radiation and then bends the rays of light that are coming from sun to further focused it on a specific point . Lenses are put on top of every solar cell by solar concentrator thus each solar cell gathers more focused light making the cells more efficient. Solar Concentrator is termed as solar concentrator as it concentrates the solar energy incident over a large surface onto a smaller surface. Suitable reflecting or refracting elements are used to achieve this concentration level, sun's virtual motion is important factor that is required to get the maximum concentration. An accurate focusing device is also required. . Solar concentrator is advantageous in the sense that solar cells can be spaced farther so now fewer cells need to be deployed, which ultimately leads to decrease in cost. Solar concentrator is a device that allows the collection of sunlight from a large area and focusing it on a smaller receiver or exit. Solar power is generated by concentrated solar power systems (CSP) by using mirrors or lenses to concentrate a large area of sunlight onto a small area, when the concentrated light is converted to heat results in the generation of electricity.

Working of this system will work same as seen with previous LI-FI technology in which photodiodes were used but now here solar panel will act as broadband receivers, the encoded data will be fed from lamp driver to led lamp, which will further pass the data in the digitalized form to the solar cells. Solar cells are the self-sufficient in the sense they will capture energy as well as transmit data, which is the advantage of this system. Solar cells will convert the incoming sunrays into electricity, so continuously energy will be captured and on the other hand, data will be transmitted. Moreover, the system also consists of solar concentrator, which is placed above solar panel. This will increase the output power of solar panel by more than half times so as the intensity of solar panel is increased more data can be transmitted for longer duration of time.

A solar concentrator used in the system is called as Fresnel solar concentrator as it employs Fresnel lenses for its working, Fresnel lenses bends the rays of light to direct the large area of sunlight it has taken towards a specific spot. Same 'principle is used by lot of people where they use a magnifying lens to focus the Sun's rays on paper to ignite fire. Shape of Fresnel lens is generally like a dashboard or rectangular having concentric rings of prisms around a lens that acts as a magnifying glass. All of these parameters let the Fresnel lens to focus scattered light from the Sun into a tight beam. Solar cells become very efficient as each of the lenses is put on every solar cell by the solar concentrators, when there is a single source of light at that point of time the concentrator tends to works best. This is possible when the Sun is a single light source.

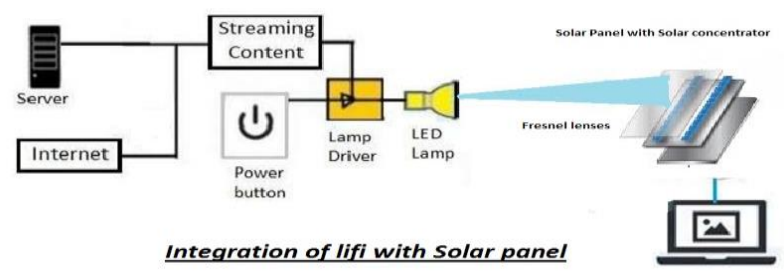

Figure 1. Li-Fi with Solar Concentrator 


\section{ADVANTAGES}

The proposed which is included will have lot of advantages over the existing system, earlier when only solar panel was used the intensity wouldn't be so high but now after putting solar concentrator which provides $45 \mathrm{Kw}$ of thermal energy at the rate of $73 \%$ efficiency, this is possible due to the reflectivity of material and highly efficient absorber. Cost of the system will be also less as fewer cells will only require as discussed earlier. Apart from this solar concentrator has lot of advantages which includes the increase of the intensity of solar panel as the energy that was available over a large surface is now possible to be concentrated onto a smaller surface., the heat loss area is also reduced due to the concentration of small area and also It ultimately helps in cost reduction as less expensive refracting medium is used instead of costlier one.

\section{Disadvantages}

As per the earlier discussion though integrating LI-FI with solar panel has lot of merits but some pitfalls may be there, the most important is that the solar panel will be inefficient to use in night time or during cloudy time s sun rays the medium for generating electricity and further transmission of data.

\section{CONCLUSION}

The proposed System consisting of solar panel and solar concentrator will increase the output power of Solar panel by more than $50 \%$. Solar concentrator which provides $45 \mathrm{Kw}$ of thermal energy at the rate of $73 \%$ efficiency, Thus for solar will work more efficient in storing energy and transmitting data for longer hours which will ultimately enhance the overall LI-FI technology.

\section{FUTURE SCOPE}

Considering the proposed system, that I have chosen uses solar panel integrated with Fresnel lens solar concentrator to increase output power of solar panel. Moreover solar panel will efficiently store solar energy that can be also be used in nighttime. Grids can be used for storing solar energy. This is possible using net-metering .In net metering, the surplus solar electricity, is send to the grid and which rolls the electric meter backwards. So at the night when system is able to produce electricity, that at that time electricity can be pulled from the grid and electric will now start rolling in forward direction.

\section{REFERENCES}

[1] D. Tsonev, S. Videv, and H. Haas, light Fidelity (Li-Fi): Towards All optical Networking, Dec. 2013

[2] Neha Singh, Divya Chauhan, Deepika Dubey Li-Fi (Light Fidelity)-The future technology In Wireless Communication International Journal of advances in computing communications, vol 1, 2013

[3] N.Navyatha, T.M.Prathyusha, V.Roja, M.Mounika Li-Fi (Light delity)-LED Based Alternative International Journal of Scientc Engineering Research, Volume 4, Is-sue 5,May-2013 1039 ISSN 22295518

[4] Mr.Shailendra Yadav,Mr.Pradeep Mishra,Miss.Minakshee Velapure,\&Prof.P.S.Togrikar,LI-FI Technology for Data Transmission through LED,IJIR, Vol-2, Issue-6, 2016 ISSN: 2454-1362
[5] Bhavya.R,Lokesh.M.R, A Survey on Li-Fi Technology, ABHIYANTRIKI: An International Journal of Engineering \& Technology 7 Volume 3, Number 1, January, 2016

[6] Wael Mahmoud Sayed Sayed Ahmed1 and Dr. Amin Babiker A/Nabi Mustafa, International Journal of Engineering, Applied and Management Sciences Paradigms, Vol. 39, September 2016 An Indexed and Referred Journal ISSN : 2320-6608

[7] Principles of LED Light Communications: Towards Networked LiFi,Svilen Dimitrov, Harald Haas, ISBN: 1107049423, 2015-0420

[8] X. Li, R. Zhang, and L. Hanzo, ooperative Load Balancing in Hybrid Visible Light Communications and WiFi,IEEE Transactions on Communications, vol. 63, no. 4,pp. 1319329, April 2015.

[9] A. Farid and S. Hranilovic ,Capacity bounds for wireless optical intensity channels with Gaussian noise,IEEE Trans. Inf. Theory, vol. 56, no. 12 pp. 6066-6077 Dec. 2010.

[10] S. Rajbhandari H. Chun G. Faulkner K. Cameron A. V. N. Jalajakumari R. Henderson et al. High-speed integrated visible light communication system: Device constraints and design considerations,IEEE J. Sel. Areas Commun. vol. 33 no. 9 pp. 1750-1757 Sep. 2015.

[11] F. Muhammad-Sukki, R. Ramirez-Iniguez, S.G. McMeekin, B.G. Stewart \& B. Clive, Solar Concentrators, International Journal of Applied Sciences (IJAS), Volume (1): Issue (1)

[12] X. Li, R. Zhang, and L. Hanzo, ooperative Load Balancing in Hybrid Visible Light Communications and WiFi,IEEE Transactions on Communications, vol. 63, no. 4,pp. 1319329, April 2015.

[13] U.suganya1, c.subhalakshmipriya, li-fi (light fidelity) technology, international journal of research in computer applications and robotics, vol.3 issue.1, pg.: 26-32 january 2015

[14] http://www.electroschematics.com/8280/maximizing-solar-panelefficiency-and-output-power.

[15] http://purelifi.com/what_is_li-fi.

[16] http://www.lifi-centre.com.

[17] http://purelifi.com

[18] http://purelifi.com/lifi-and-solar-panels-close-the-digital-divide-lifi-atted-wired-magazine.

[19] https://www.youtube.com/watch?v=iHWIZsIBj3Q

[20] http://mechanicalinventions.blogspot.in/2014/07/classification-of-solarconcentrators-advantages.html.

[21] http://www.homepages.ed.ac.uk/hxh/LiFi_PAPERS/what_is_LiFi_invite d_jlt_ecoc15.pd.

\section{AUTHOR PROFILE}

Siddharth Agarwal is a Bachelor of Technology in the Information Technology Engineering Department at the Bharati Vidyapeeth Deemed University College of Engineering. His research interests include Networking.

Yash Omer is a Bachelor of Technology in the Information Technology Engineering Department at the Bharati Vidyapeeth Deemed University College of Engineering. His research interests include kernel/Wireless Networking.

T.B. Patil is Assistant Professor in the Information Technology Engineering Department at the Bharati Vidyapeeth Deemed University College of Engineering. His research interests include Networking, Computer Graphics.
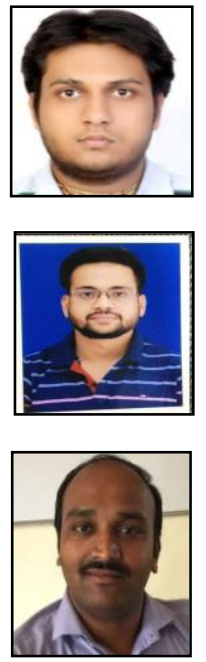
Supriya C. Sawant is Associate Professor in the Electronics \& Telecommunication Engineering Department at the B Smt. Kashibai Navale College of Engineering. Her research interests include signal processing.

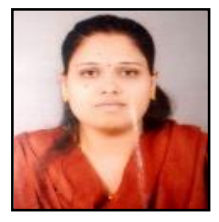
(http://creativecommons.org/licenses/by/4.0/). 